\title{
A Case of Selective Straight Sinus Thrombosis (SST): Sudden Onset and Evolution
}

\author{
Sergiacomi G, Pugliese L*, Cola G, Sancesario G \\ and Floris $\mathbf{R}$ \\ Department of Diagnostic Imaging, Molecular Imaging, \\ Interventional Radiology and Radiation Therapy, \\ University of Rome Tor Vergata, Italy \\ *Corresponding author: Luca Pugliese, Department \\ of Diagnostic Imaging, Molecular Imaging, Interventional \\ Radiology and Radiation Therapy, PTV Foundation, "Tor \\ Vergata" Hospital, University of Rome "Tor Vergata", \\ Viale Oxford, 81- 00133, Roma, Italy
}

Received: November 02, 2018; Accepted: December 27, 2018; Published: December 31, 2018

\begin{abstract}
Cerebral venous thrombosis, thrombosis of the intracranial veins and sinuses, is a rare type of cerebrovascular disease that hits about 0,1-0,2 people per 100000 and accounts for $0.5 \%$ of all stroke. The mortality range from 5 to $15 \%$. Straight sinus thrombosis is a rare type of cerebral venous sinus thromboses and is extremely difficult to diagnose, especially at its acute stage. The diagnosis is often delayed in many cases of straight sinus thrombosis that brings to treatment delay and a poor prognosis. We illustrate a case of a patient with straight sinus thrombosis with bilateral hemorrhage lesions who showed a fast evolution and sudden onset.

Keywords: Cerebral venous sinus thrombosis; Straight sinus; D-dimers; TC; MRI
\end{abstract}

\section{Abbreviations}

CVT: Cerebral Venous Thrombosis; CT: Computed Tomography; MRI: Magnetic Resonance Imaging; SST: Straight Sinus Thrombosis

\section{Introduction}

Cerebral Venous Thrombosis (CVT), thrombosis of the intracranial veins and sinuses, is a rare type of cerebrovascular disease that hits about $0,1-0,2$ people per 100000 [1] and accounts for $0.5 \%$ of all stroke. The mortality range from 5 to $15 \%$ [2]. CVT predominantly affects adults younger than 50 years and children, and less than $10 \%$ interest's patients older than 65 years [3].

The main symptoms vary from mild headache to severe intracranial bleeding and ischemic infarction $[4,5]$.

Straight Sinus Thrombosis (SST) is a rare type of cerebral venous sinus thromboses and is extremely difficult to diagnose, especially at its acute stage. The diagnosis is often delayed in many cases of SST that brings to treatment delay and a poor prognosis.

\section{Case Report}

A 67-year-old man was admitted to another Hospital because of strong headache and dizziness. The patient's medical history was unremarkable except for a deep venous thrombosis seven years ago on medical therapy with rivaroxaban, stopped one month before this event for unexplained reasons. An initial plain CT scan was performed. It showed hemorrhagic focal area in the left temporal occipital lobe. The day after the patient was discharged with the suggestion to perform MRI. But gradually the patient got worsen with disturbed consciousness. On admission to our Hospital, the patient was sleepy but easily re-waken by call, eyes movements and pupillary reflexes were normal; he was unable to raise his legs from the bed but he moved that after pain, bilateral plantar reflex was normal. Laboratory studies were normal except for an increase of $\mathrm{D}$-dimer to $7596,00 \mathrm{ng} / \mathrm{mL}(0,00-500,00)$.

The CT (Computed Tomography) scan confirmed unchanged the bleeding in the occipital lobe and also demonstrated a focal hemorrhagic nucleus in the nucleus-capsular of the left lobe (Figure $1)$.

Then an MRI (magnetic resonance imaging) study was performed. On T2-weighted demonstrated bilateral and symmetrical hyperintensity areas of altered signal in the context of the putamen and pale globes bilaterally (Figure 2).

Fast Field Echo (FFE) sequences documented altered areas in whose context there are widespread hemoglobin catabolism's derivate in various stages of evolution in time with the extension to the white substance periventricular and peri-ependimal bilaterally and in the splenius of the corpus callosum (Figure 3).

DWI documented bilateral hyper-intense signals (Figure 4).

Basing on MRI differential diagnosis of bilateral lesions include tumors, metabolic or vascular lesions. Afterwards a CT with contrast medium was repeated and a venous acquisition phase was performed that showed a filling defect of straight and left transvers sinus, like thrombosis without arterial pathological stenosis (Figure 5). These findings confirmed the diagnosis. Suddenly he started medical therapy with heparin.

During the following days, his neurological state gradually got worsen.

Unfortunately, the patient developed an acute respiratory failure with a pneumonia and he was transferred to the intensive care.

$\mathrm{He}$ also presented C677T mutation in the MethyleneTetrahydrofolate Reductase Gene (MTHFR) and in factor V of Leiden, both in heterozygosity.

\section{Discussion}

Cerebral venous and sinus thrombosis is a rare cause of stroke and has a wide range of presentations. Early diagnosis and therapy can be lifesaving. The superior sagittal sinus and the transverse sinus are the most frequent sites of thrombosis, involved in 62-80 and 


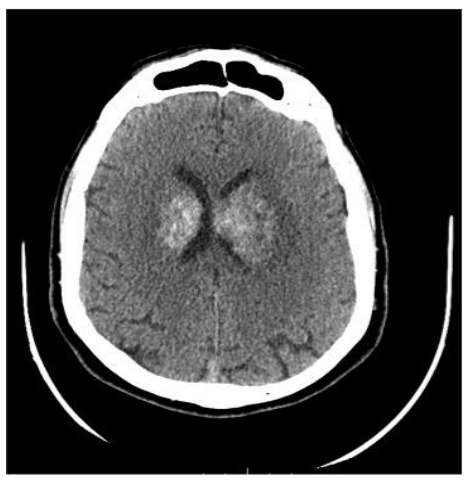

Figure 1: CT scan in axial plane which demonstrated bleeding in the occipital lobe and a focal hemorrhagic nucleus in the nucleus-capsular of the left lobe.

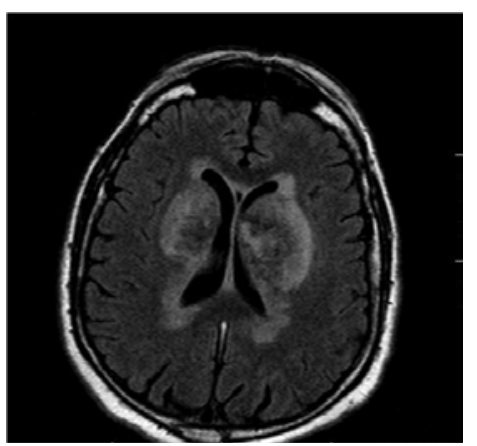

Figure 2: MRI scan T2-weighted which demonstrated bilateral and symmetrical hyper-intensity areas of altered signal in the context of the putamen and pale globes bilaterally.

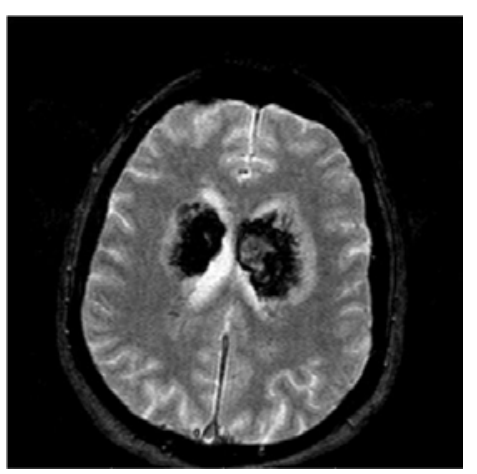

Figure 3: MRI scan FFE documented altered areas in whose context there are widespread hemoglobin catabolism's derivate in various stages of evolution in time with the extension to the white substance periventricular and peri-ependimal bilaterally and in the splenius of the corpus callosum.

$38-86 \%$ of cases [6-8]. So that, bilateral thalamic infarction is a rare manifestation of dural sinus thrombosis.

CVT is typically multifactorial, which means that the identification of a risk factor or even a cause would not deter clinicians from looking for other risk factors, particular congenital thrombophilia $[9,10]$. The $44 \%$ of the patients had more than one cause or predisposing factor, and congenital or hereditary thrombophilia was present in $22 \%$ of patients. So, among risk factors, hereditary thrombophilia coincide with those for more common sites VTE, while others, such as head

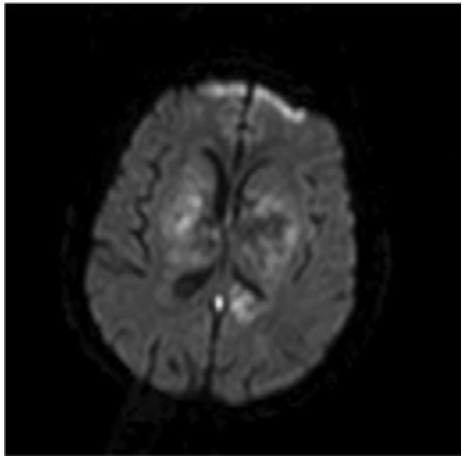

Figure 4: MRI scan DWI documented DWM bilateral hyper-intense signals.

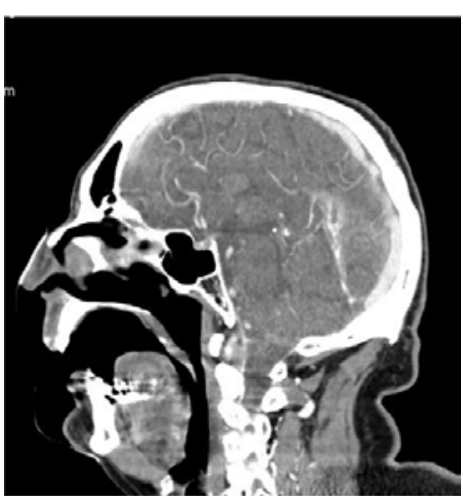

Figure 5: TC scan with iodinate contrast in sagittal plane that showed a filling defect of straight and left transvers sinus.

trauma and regional infections, are specific to CVT [4,11].

Others different risk factors have been associated with CVT in addition to those mentioned above like cancer [12,13], myeloproliferative neoplasms [14], systemic lupus erythematosus [15-17], antiphospholipid syndrome [18-20], sex-specific like oral contraceptives [21,22], Pregnancy and puerperal infections [23], and dehydration [24].

The diagnosis of CVST is obscured because the wide variety of symptoms, which range from altered sensorium, focal neurological signs and seizures to symptoms i.e. raised intracranial pressure such as vomiting and blurring vision. In all series, headache is present in over $80 \%$ of cases and it is the most common initial symptom too. Furthermore, a cause cannot be found in about $15 \%$ of cases [5]. The possibility of isolated headache, as the only symptom of CVT has recently been emphasized. The individual outcome may still be difficult to predict, and the disorder may occasionally worsen despite anticoagulation.

Because of the wide variety of aspects in presenting symptoms of CVT, would be of great practical interest to have a test that would be easy to do in emergency care and would easily rule out CVT. Several studies tested the value of $\mathrm{D}$-dimer measurements because in deep venous thrombosis of the legs, low values $(<500 \mathrm{ng} / \mathrm{mL})$ have a high negative predictive value [25]. Indeed, in most patients with recent CVT, D-dimer concentrations are increased. So that, a negative D-dimer assay may make the diagnosis of CVT very unlikely. Thus, a 
negative D-dimer assay cannot rule out CVT in the setting of a recent isolated headache.

MRI venography and CT venography are both adequate for the diagnosis of CVT but the former is clearly superior for the greater spatial and anatomical resolution. CT venography gives a good visualization of the major dural sinuses, is readily available, can be used for patients who have contraindications to MRI and is quicker than MRI. It can demonstrate density in thrombosed venous sinus. Its use may be limited because of low resolution of the deep venous system and cortical veins. The sign of acute CVT is an increased attenuation of the occluded sinus. CT venography is more sensitive to low blood flow than is Time of Flight (TOF) magnetic resonance venography, which tends to overestimate the degree of thrombosis in cases of partial vessel occlusion. In conclusion, CT venography is as accurate as MR venography for diagnosing cerebral Seno-venous thrombosis. Unfortunately, our patient got worsen and so he was unable to perform a MRI venography acquisition.

The aims of antithrombotic treatment in CVT are: 1) to recanalize the occluded sinus/vein, 2) to combat the propagation of the thrombus, 3) to prevent pulmonary embolism, 4) to treat the underlying pro-thrombotic state

The clinical course of CVT is unpredictable in the first days after diagnosis, and about one quarter of patients deteriorate in that phase. Death in the acute phase happens in $4 \%$ of patients and, as mentioned previously, is generally due to transtentorial herniation $[9,26,27]$. Status epilepticus and medical complications, such as sepsis and pulmonary embolism are other possible causes of early death in patients with CVT. First-line treatment for CVT is heparin, even in the presence of an intracerebral hemorrhage. In a trial completed in 2017, endovascular therapy did not improve the clinical outcome of patients with severe CVT [28].

\section{Conclusion}

CVT thus remains a diagnostic and therapeutic challenge. Straight sinus thrombosis is a rare form of cerebral venous thrombosis and is extremely difficult to diagnose. A diagnosis of CVT is very often neglected or delayed, given the extreme variability of clinical symptoms and findings at neuroimaging. Thrombosis of deep cerebral veins is a very rare, poorly documented, and potentially fatal condition. The presence of bilateral brain lesions with an acute onset of symptoms and a suggested anamnestic history are suggestive for CVT.

\section{References}

1. Devasagayam S, Wyatt B, Leyden J, Kleinig T. Cerebral venous sinus thrombosis incidence is higher than previously thought: a retrospective population- based study. Stroke. 2016; 47: 2180-2182.

2. Coutinho JM, Zuurbier SM, Stam J. Declin-mortality in cerebral venous thrombosis: a systematic review. Stroke. 2014; 45: 1338-1341.

3. Ferro JM, Canhão P, Bousser MG, Stam J, Barinagarrementeria F, ISCVT Investigators. Cerebral vein and dural sinus thrombosis in elderly patients. Stroke. 2005; 36: 1927-1932.

4. Stam J. Thrombosis of the cerebral veins and sinuses. N Engl J Med. 2005; 352: 1791-1798.

5. Coutinho JM, Stam J, Canhão P, Barinagarrementeria F, Bousser MG, Ferro $\mathrm{JM}$, et al. Cerebral venous throm-bosis in the absence of headache. Stroke.
2015; 46: 245-247.

6. Wall M, Corbett JJ. Revised diagnostic criteria for the pseudotumor cerebri syndrome in adults and children. Neurology. 2014; 83: 198-199.

7. Damak M, Crassard I, Wolff $\mathrm{V}$, Bousser MG. Isolated lateral sinus thrombosis: a series of 62 patients. Stroke. 2009; 40: 476-481.

8. Kumral E, Polat F, Uzunköprü C, Callı C, Kitiş Ö. The clinical spectrum of intracerebral hematoma, hemorrhagic infarct, non-hemor- rhagic infarct, and non-lesional venous stroke in patients with cerebral sinus-venous thrombosis. Eur J Neurol. 2012; 19: 537-543.

9. FerroJM, CanhãoP, StamJ, Bousser MG, Barinagarrementeria F, ISCVT Investigators. Prognosis of cerebral vein and dural sinus thrombosis: results of the International Study on Cerebral Vein and Dural Sinus Thrombosis (ISCVT). Stroke. 2004; 35: 664-670.

10. Dentali F, Poli D, Scoditti U, Di MN, De Stefano V, Siragusa S, et al. Cerebral VE in Thrombosis International Study Investigators. Long-term outcomes of patients with cerebral vein thrombosis: a multicenter study. J Thromb Haemost. 2012; 10: 1297-1302.

11. Coutinho JM. Cerebral venous thrombosis. J Thromb Haemost. 2015; 13 : S238-S244.

12. Couturier MA, Huguet F, Chevallier P, Suarez F, Thomas X, Escoffre-Barbe $M$, et al. Cerebral venous thrombosis in adult patients with acute lymphoblastic leukemia or lymphoblastic lymphoma during induction chemotherapy with I-asparaginase: The GRAALL experience. Am J Hematol. 2015; 90: 986-991.

13. Zuurbier SM, Lauw MN, Coutinho JM, Majoie CB, van der Holt B, Cornelissen $\mathrm{JJ}$, et al. Clinical course of cerebral venous thrombosis in adult acute lymphoblastic leuke- mia. J Stroke Cerebrovasc Dis. 2015; 24: 1679-1684.

14. Dentali F, Ageno W, Rumi E, Casetti I, Poli D, Scoditti U, et al. Cerebral venous thrombosis and myeloproliferative neoplasms: results from two large databases. Thromb Res. 2014; 134: 41-43.

15. Vidailhet M, Piette JC, Wechsler B, Bousser MG, Brunet P. Cerebral venous thrombosis in systemic lupus erythematosus. Stroke. 1990; 21: 1226-1231.

16. Wang L, Chen H, Zhang Y, Liu W, Zheng W, Zhange X, et al. Clinical characteristics of cerebral venous sinus thrombosis in patients with systemic lupus eryth- ematosus: a single-centre experience in China. J Immunol Res. 2015; 2015: 540738 .

17. Wysokinska EM, Wysokinski WE, Brown RD, Karnicki K, Gosk-Beirska I, Grill D, et al. Thrombophilia differences in cerebral venous sinus and lower extremity deep venous thrombosis. Neurology. 2008; 70: 627-633.

18. Narayan D, Kaul S, Ravishankar K, Suryaprabha T, Bandaru VC, Mridula $\mathrm{KR}$, et al. Risk factors, clinical profile, and long-term outcome of 428 patients of cerebral sinus venous thrombosis: insights from Nizam's Institute Venous Stroke Registry, Hyderabad (India). Neurol India. 2012; 60: 154-159.

19. Christopher R, Nagaraja D, Dixit NS, Narayanan CP. Anticardioli- pin antibodies: a study in cerebral venous thrombosis. Acta Neurol Scand. 1999; 99: 121-124.

20. de Bruijn SF, Stam J, Koopman MM, Vandenbroucke JP. The Cerebral Venous Sinus Thrombosis Study Group. Case-control study of risk of cerebral sinus thrombosis in oral contraceptive users and in [correction of who are] carriers of hereditary prothrombotic conditions. BMJ. 1998; 316: 589-592.

21. Martinelli I, Sacchi E, Landi G, Taioli E, Duca F, Mannucci PM. High risk of cerebral-vein thrombosis in carriers of a prothrombin- gene mutation and in users of oral contraceptives. N Engl J Med. 1998; 338: 1793-1797.

22. Cantú C, Barinagarrementeria F. Cerebral venous thrombosis associated with pregnancy and puerperium. Review of 67 cases. Stroke. 1993; 24: 1880 1884.

23. deVeber G, Andrew M, Adams C, Bjornson B, Booth F, Buckley DJ, et al. Canadian Pediatric Ische- mic Stroke Study Group. Cerebral sinovenous thrombosis in children. N Engl J Med. 2001; 345: 417-423.

24. Kosinski CM, Mull M, Schwarz M, Koch B, Biniek R, Schlafer J, et al. Do normal D-dimer levels reliably exclude cerebral sinus thrombosis?. Stroke. 2004; 35: 2820-2825. 
25. Canhao P, Ferro JM, Lindgren AG, Bousser MG, Stam J, Barinagarrementeria $F$, et al. Causes and predictors of death in cerebral venous thrombosis. Stroke. 2005; 36: 1720-1725.
26. Dentali, F, Gianni M, Crowther MA, Ageno W. Natural history of cerebral vein thrombosis: a systematic review. Blood. 2006; 108: 1129-1134.

27. US National Library of Medicine. Clinical Trials. 2017
Austin J Radiol - Volume 5 Issue 2 - 2018

ISSN : 2473-0637 | www.austinpublishing group.com

Pugliese et al. () All rights are reserved
Citation: Sergiacomi G, Pugliese L, Cola G, Sancesario G and Floris R. A Case of Selective Straight Sinus Thrombosis (SST): Sudden Onset and Evolution. Austin J Radiol. 2018; 5(2): 1085. 